\title{
Approche de Gestalt-thérapie des expériences dépressives
}

\author{
Gianni Francesetti \& Jan Roubal
}

\author{
Psychotherapie-Wissenschaft 10 (2) 46-48 2020 \\ www.psychotherapie-wissenschaft.info \\ CC BY-NC-ND \\ https://doi.org/10.30820/1664-9583-2020-2-46
}

Mots clés : Gestalt-thérapie, dépression, deuil, psychopathologie, fonctions propres

Cet article place l'expérience dépressive dans un cadre issu d'une perspective de Gestalt-thérapie phénoménologique. Il se concentre sur la manière dont le patient et le thérapeute sont affectés en même temps par un champ dépressif, et il propose une approche radicalement relationnelle de la dépression, dans laquelle le client et le thérapeute sont vus comme victimes de dépression, et maintenant dans la situation de thérapie. Afin de mieux mettre en exergue le cœur de la souffrance de personnes en dépression et la spécificité du traitement de cette dernière, on commence par distinguer le deuil et l'expérience dépressive.

Le deuil est perçu comme une manière d'assimiler une perte importante. Il aide l'individu non seulement à assimiler la perte subie, mais aussi son expérience relationnelle avec l'être perdu. L'un des dons que nous fait la mort est celui de nous révéler la beauté de l'être disparu. Son absence révèle la profondeur et la valeur de sa présence. Le côté négatif de cela pour ceux qui restent est la découverte que ceux-ci font de leurs propres absences en présence de l'être aimé : «Pourquoi n'ai-je pas réalisé combien il était important, merveilleux et enrichissant d'être avec luilelle? " La période de deuil sert à établir une double loyauté : la loyauté envers la relation qui a été perdue, et la loyauté envers la vie qui doit continuer. Lorsque cette double loyauté a été instaurée, la période de deuil prend fin. Le deuil permet à la vie de préserver la richesse dont on hérite de la relation passée, et de se lancer une fois de plus dans la fontaine éternellement jaillissante de la nouveauté.

Pendant le réveil immédiat d'un deuil, c'est le caractère irrémédiable qui tend à dominer l'avant-plan, les souvenirs du passé tendant à remonter à la surface, la prise de conscience d'avoir été avec l'être perdu et de tout ce que vous avez vécu avec lui. De cette manière, l'expérience de la relation est assimilée et le sujet atteint progressivement un état de présence dans l'absence. Celui ou celle qui reste apprend à porter l'être perdu avec lui ou elle, en développant une nouvelle capacité d' "être-avec » l'autre et une nouvelle forme de fidélité. Une expérience similaire peut être observée avec d'autres types d'événements importants de la vie comprenant des pertes, comme l'avortement, le licenciement, le fait de devenir un réfugié, etc. Le processus d'assimilation requis est semblable à celui qui est demandé dans les cas de deuil.
Dans l'expérience du deuil, une personne ou un objectif spécifique devient hors d'atteinte et c'est là que réside la perte subie. L'expérience de la dépression mélancolique se présente sous un autre jour : ce qui est perdu est ce qui ancre le sujet dans le tissu qui le relie au monde. Dans le premier cas, quelqu'un perd l'autre personne à laquelle il est attaché, dans le second cas, on perd les conditions qui permettent de former un tel lien. La gravité de la dépression d'un patient peut être mesurée dans les formes que prend le détachement de l'entre-deux, qui est le tissu qui nous relie au monde et à la vie à tous les instants. Dans les cas de mélancolie, ce terrain commun a cessé d'exister et ne peut par conséquent plus être traversé. Cet entredeux n'est plus un lieu de rencontre. Il s'est au contraire transformé en un abîme cosmique insurmontable.

Dans de telles conditions, la fonction de l'ego est potentiellement réduite à néant, à un état de stupeur dans lequel rien ne se passe. La fonction id (la fonction préréflexive qui nous relie au monde en précédant le fossé entre le moi et le monde, l'organisme et l'environnement), assume à sa place un rôle plus significatif. On est en présence d'un désordre de l'id de la situation dans lequel est générée la source même de la subjectivité, du temps, de l'espace et de l'intentionnalité - la vie elle-même. Le profond dysfonctionnement de la fonction id signifie qu'il est devenu impossible de co-créer une figure de contact. Ce dysfonctionnement se trouve au cœur de la difficulté qu'éprouve le thérapeute à établir une relation avec le patient, en assurant les allers et les retours habituels de résonances, consonances et dissonances susceptibles d'emplir l'espace et le temps thérapeutiques. En bref, rien ne se répercute dans l'entre-deux thérapeutique.

L'expérience dépressive est l'expression d'un champ relationnel. Le temps et l'espace sont les routes que nous concevons de nous-mêmes lorsque que nous frayons notre chemin vers ce qui est aimé et nécessaire. Ce sont des variables dépendant de la relation, générées par les impulsions du voyage lui-même, qui n'est jamais seulement un simple mouvement mais toujours un co-mouvement. Si ce mouvement fait défaut, ce dont nous faisons l'expérience est l'abîme qui nous sépare. Le pont affectif sur lequel nos mois eux-mêmes se constituent et duquel jaillit la subjectivité est perdu. Les expériences dépressives sont l'expression en l'individu d'une expérience relationnelle 
spécifique : à savoir l'impossibilité d'atteindre l'autre. En termes plus précis : la dépression est la manière dont le sujet fait l'expérience de l'abandon de tout espoir face à l'inefficacité de ses vaines tentatives pour atteindre l'autre. La dépression peut par conséquent être vue comme l'absence de présence, et peut être comprise comme un phénomène relationnel co-construit présentant trois caractéristiques intrinsèques et essentielles : un profond attachement, dans lequel l'autre est aimé et nécessaire, l'échec de tous les efforts pour atteindre l'autre et l'absence émotionnelle de l'autre de la relation.

La réaction habituelle de la famille du patient/de la patiente ou des personnes les plus proches et les plus chères vis-à-vis de l'état dépressif de ce dernier ou de cette dernière est polaire. Elle commence par chercher à l'encourager ("Allez, tout va bientôt s'arranger. Allons un peu nous divertir, cela t'aidera à surmonter cette situation »). Plus tard, lorsque cet effort s'avère vain et qu'elle commence à s'y épuiser, elle s'efforce de se protéger et s'éloigne de la personne déprimée (souvent avec une agressivité plus ou moins dissimulée). Le ou la thérapeute se retrouve dans le même schéma relationnel et tend à ressentir des impulsions qui l'amènent à répéter les réactions décrites vis-à-vis de la personne dépressive. Le ou la thérapeute se sent attiré(e) par l'expérience de cette dernière comme par un aimant. Puis il ou elle adopte une distance émotionnelle de sécurité en arborant un masque professionnel, comme si l'expérience dépressive ne lui était pas familière, en commençant parfois à assumer une responsabilité inappropriée de toute la situation. Ou bien le ou la thérapeute devient plus proche en partageant dans une certaine mesure l'expérience dépressive du ou de la patiente. Les échecs répétés des expériences du ou de la thérapeute mènent ce dernier ou cette dernière à la solitude, au désemparement, à la honte, et commencent à lui peser. Dans ce cas, il ou elle peut se sentir menacé(e) par le risque d'une " contagion dépressive ", il ou elle ressent que : "C'est trop pour moi! " et réagit en se protégeant lui- ou elle-même et/ou en devenant agressif vis-à-vis du ou de la patiente : "Il ou elle est insupportable. Il ou elle a besoin de moi et je suis là, lui tendant la main, mais il ou elle est incapable de s'en apercevoir! » ou bien encore "Rien de ce que je fais n'est d'une quelconque utilité, il ou elle fait tout à sa guise et c'est tout! » Le ou la thérapeute peut être alors tenté(e) de défier le ou la patiente : "Et bien c'est entendu, voyons ce qui aura le plus de poids : mon engagement ou ton inertie!»

Grâce à sa conscience de la situation, le ou la thérapeute garde la possibilité de sortir de ce schéma relationnel rigide et répond différemment à la personne déprimée - il ou elle reste disponible pour le contact, ne s'adresse plus de reproches ni au patient ou à la patiente, sans pour autant abandonner tout espoir. Ce faisant, le ou la thérapeute change l'organisation rigide de l'environnement, tout en ouvrant un espace permettant à son patient ou à sa patiente de changer. Il est important pour le ou la thérapeute d'être conscient(e) de sa propre expérience et de ne pas reprocher la situation à son patient/sa patiente ou à soi-même, parce que des reproches représentent une caractéristique distincte d'organiser un champ dépressif. La tâche du thérapeute consiste à rester présent(e), alors qu'il serait facile de se perdre, de s'endormir ou de perdre son sang-froid, sans devenir dépressif quand il est si facile de perdre tout espoir. Une telle situation représente une des tâches les plus ardues que doit affronter un ou une psychothérapeute : il ou elle se met soi-même à la disposition du patient ou de la patiente, mais dans cette situation sa fonction id rencontre un abîme. Comment il ou elle peut habiter une telle cavité, un tel abîme ?

Le ou la thérapeute doit se remémorer de toutes ses expériences, parce que celles-ci représentent une manière d'être-avec l'autre dans le champ relationnel. La perspective du champ fournit une assistance au ou à la thérapeute à deux niveaux : elle le ou la met en capacité de donner du sens à ses émotions, tout en lui permettant d'agir. En se posant simplement la question «comment allons-nous poursuivre notre dépression ensemble maintenant? ", le ou la thérapeute restaure l'équilibre de la situation. L'expérience dépressive est comme un marais, tant pour le patient que pour le thérapeute. Il est inutile de sauter dans un marais, de se donner des buts thérapeutiques ambitieux, de se forcer à être optimiste. Plus le ou la thérapeute encourage le patient à sauter, et plus le patient s'enfonce. On a plutôt besoin de petits mouvements visant à rechercher des sources mineures d'assistance. Le ou la thérapeute ne force pas le patient à l'introspection, ne recherche pas ce qui ne marche pas. Ce schéma de rétroréflexion et d'autocritique est déjà trop profondément à l'œuvre. Le travail se concentre autour de la tâche primaire consistant à créer un environnement sauf, un champ relationnel sauf dans lequel les capacités d'auto-guérison du patient peuvent être activées.

Dans la relation thérapeutique, le thérapeute est le gardien de l'espoir mais aussi, d'une façon plus radicale, du temps et de l'espace. Le temps et l'espace thérapeutiques deviennent des loci dans lesquels le temps et l'espace de l'expérience recommencent progressivement à coaguler. Le ou la thérapeute se redonne la possibilité d'agir à travers la prise de conscience que le client et le thérapeute co-créent ensemble les événements au fur et à mesure qu'ils se déroulent. Ceci ayant été réalisé, l'action effectuée peut être quelque chose d'aussi simple que le fait de rester éveillé, que de conserver la capacité de penser ou de solliciter l'aide d'un tiers si le ou la thérapeute s'approche de l'abîme dépressif. De cette manière, le ou la thérapeute ne perd pas l'espoir de finir par atteindre le patient ou la patiente, et est capable de rester à sa disposition. Ce maintien de la capacité de garder l'espoir et de rester présent constitue le fondement même d'une thérapie visant à traiter une grave dépression.

\section{Les auteurs}

Gianni Francesetti, Dr., est un psychiatre, Gestalt-thérapeute, professeur adjoint d'approche phénoménologique et existentielle au département de psychologie de l'Université de Turin (Italie), formateur et superviseur international. Il est président de l'IPsiG - International Institute for Gestalt Therapy and 
Psychopathology and of Poiesis - Gestalt Therapy Clinical Centre of Torino (Institut international de Gestalt-thérapie, de psychopathologie et de poïétique du Centre clinique de Gestalt-thérapie de Turin).

Jan Roubal, Dr., est professeur associé à l'Université Masaryk à Brno, République Tchèque, où il travaille aussi au Centre dédié à la recherche en psychothérapie. Il travaille en tant que psychothérapeute et que psychiatre. Il a créé le Centre de formation en intégration psychothérapeutique et les Études de formation en Gestalt en République Tchèque. Il travaille également au niveau international en tant que formateur et superviseur en psychothérapie.

\section{Contact}

Gianni Francesetti

E-Mail : gianni.francesetti@gmail.com

Jan Roubal

E-Mail : jan.roubal.cz@gmail.com 